\title{
¿INCREMENTAR LA EDAD LEGAL DE JUBILACIÓN \\ Y EXTENDER LA TRAYECTORIA LABORAL? \\ APUNTES PARA LA DISCUSIÓN SOBRE EMPLEO EN PERSONAS MAYORES EN CHILE*
}

\author{
INCREASING THE FULL PENSION AGE AND EXTENDING \\ WORKING LIFE? INPUTS FOR THE DISCUSSION \\ ON EMPLOYMENT AMONG OLDER PEOPLE IN CHILE**
}

\author{
AUGMENTER L'AGE LEGAL DE LA RETRAITE ET PROLONGER \\ LA VIE ACTIVE? CONTRIBUTIONS A LA DISCUSSION SUR \\ L'EMPLOI CHEZ LES SENIORS AU CHILI
}

\author{
Ignacio MADERO-CABIB ${ }^{* * *}$ \\ Rodrigo PALOMO VÉLEZ ${ }^{* * * *}$ \\ María Soledad JOFRÉ BuSTOS ${ }^{* * * * *}$
}

\begin{abstract}
RESUMEN: Basados en evidencia empírica nacional e internacional, en el presente artículo planteamos una discusión sobre diferentes dimensiones involucradas en las políticas que buscan incentivar el empleo en personas mayores y aplazar la transición a la jubilación en Chile. En concreto,
\end{abstract}

* Recibido el 8 de enero de 2019 y aceptado para su publicación el 25 de marzo de 2019.

** Este artículo ha sido realizado en el marco de la ejecución del Proyecto Fondef ID16AM0006 "El maltrato estructural a las personas mayores en Chile. Formulación de un índice multidimensional”, y el Proyecto Fondecyt Iniciación No1118036 (ambos financiados por la Comisión Nacional de Investigación Científica y Tecnológica de Chile - Conicyt), y por el Núcleo Milenio para el Estudio del Curso de Vida y la Vulnerabilidad (financiado por la Iniciativa Milenio, Ministerio de Economía de Chile).

*** Instituto de Sociología, Facultad de Ciencias Sociales, Pontificia Universidad Católica de Chile. Núcleo Milenio para el Estudio de Curso de Vida y la Vulnerabilidad (MLIV). Correo electrónico: i.maderocabib@uc.cl.

**** Centro de Estudios de Derecho del Trabajo y de la Seguridad Social de la Universidad de Talca (Centrass). Correo electrónico: rpalomo@utalca.cl.

***** Centro de Estudios de Derecho del Trabajo y de la Seguridad Social de la Universidad de Talca (Centrass).Correo electrónico:majofre@utalca.cl.

Revista Latinoamericana de Derecho Social
Núm. 29, julio-diciembre de 2019, pp. 145-175 
en primer lugar discutimos la actual participación laboral de hombres y mujeres mayores, sus condiciones laborales, y las trayectorias laborales y de cotización a las pensiones de las personas que hoy están próximas a jubilar. En segundo término, nos referimos a la manera en que se ha abordado la extensión de las carreras laborales y el incremento de la edad legal de jubilación en diferentes países. En tercer lugar, discutimos los principales desafíos de salud que actualmente enfrentan las personas mayores en Chile. En cuarto lugar, nos referimos al contexto cultural vinculado al envejecimiento poblacional en este país. Luego de esto, en la conclusión, resumimos los aspectos discutidos y proponemos posibles orientaciones que, desde nuestro punto de vista, debería tener una política pública que busque incentivar la postergación de la jubilación.

Palabras clave: jubilación, empleo, personas mayores, Chile.

ABSTRACT: Based on national and international literature, in this manuscript we discuss different dimensions involved in policies that seek to encourage employment for older persons as well as retirement beyond full pension age. Concretely, we first discuss the current labor force participation of older men and women, their working conditions, and the occupational trajectories and pension contribution histories of individuals who are now close to the retirement age. Second, we refer to the ongoing discussion on the extension of working life and the delay of the full pension age in different countries. Third we discuss the main health challenges that older people in Chile currently face. Fourth, we refer to the cultural context associated with older individuals in this country. Finally, in the conclusion, we summarize the discussed aspects through the manuscript and suggest orientations that, from our point of view, should consider a public policy that seeks to encourage later retirement.

Keywords: retirement, employment, older people, Chile

RESUME: À partir d'une révision exhaustive de la littérature nationale et internationale, ce manuscrit problématise et discute différentes dimensions des politiques visant à encourager l'emploi des personnes âgées ainsi que la retraite au-delà de l'âge légal de la retraite. Concrètement, nous discutons tout d'abord la participation actuelle au marché du travail des hommes et des femmes seniors, leurs conditions de travail, ainsi que les trajectoires professionnelles et l'histoire des cotisations de pension d'individus qui sont proches à la retraite. Deuxièmement, nous nous référons au débat en cours dans plusieurs pays sur l'extension de la vie active et le repoussement de l'âge de la retraite. Nous aborderons ensuite 
les principaux défis de santé auxquels sont confrontées les personnes âgées au Chili. Quatrièmement, nous faisons référence au contexte culturel associé aux personnes âgées dans ce pays. Enfin, dans la conclusion, nous résumons les aspects discutés dans le manuscrit et proposons des orientations qui, de notre point de vue, devraient envisager une politique publique visant à encourager la retraite tardive.

Mots-clés: retraite, emploi, seniors, personnes âgées, Chili.

SumARIO: I. Introducción. II. Contexto laboral y de pensiones de las personas mayores en Chile. III. La tendencia hacia la extensión de las trayectorias laborales en diferentes países. IV. La dimensión de salud de las personas mayores en Chile. V. Dimensión cultural de las personas mayores en Chile. VI. Discusión y conclusión.

\section{INTRODUCCIÓN}

D urante las últimas tres décadas, Chile ha experimentado una transición demográfica sin precedentes como consecuencia del aumento de la esperanza de vida y la disminución de las tasas de natalidad. Mientras que en 1990 el 9\% de la población tenía más de sesenta años, actualmente este grupo etario representa el 16\% de la población total, y se espera que alcance el 25\% en 2035, y, además, que dicho año supere a la población menor a quince años. ${ }^{1}$

El aumento sustantivo en la esperanza de vida, así como el decrecimiento del número de nacimientos, han conducido (y seguirá conduciendo) a diferentes desafíos para la sociedad. Uno de los más evidentes corresponde a la sostenibilidad financiera del sistema de pensiones en la vejez. En Chile, ni los diseñadores ni los responsables administrativos del actual esquema de pensiones de capitalización individual anticiparon adecuadamente la necesidad de que el ahorro de cotizaciones previsionales tuviera que solventar periodos de vida cada vez más extensos, luego de la transición a la jubilación. Según cifras de la Organización para la Cooperación y el Desarrollo Económicos

1 Bravo, David y Hughes, E., Encuesta calidad de vida del adulto mayor e impacto del pilar solidario, Centro UC Encuestas y Estudios Longitudinales, 2018. Véase también: CEVE UC, Trabajo y personas mayores en Chile. Lineamientos para una politica de inclusión laboral, Santiago de Chile, Pontificia Universidad Católica de Chile, 2018. 
(OECD), la edad efectiva de jubilación de las mujeres en este país es en promedio 67.2 años, y 71 años para los hombres. Considerando que la expectativa de vida en Chile actualmente es en promedio 86.6 años para las mujeres y 84.6 años para los hombres, la duración promedio de la jubilación en mujeres es de 19.4 años, y en hombres 13.6 años.

Atendiendo a este contexto, en 2014 el gobierno de la presidenta Michelle Bachelet mandató a una Comisión Asesora ${ }^{2}$ que identificara las debilidades existentes del sistema de pensiones y sugiriera medidas políticas para superarlas. Los análisis de la Comisión indicaron tres limitaciones principales del sistema de pensiones: bajos ingresos de pensiones, bajas tasas de reemplazo (esto es, el porcentaje que representa la pensión del promedio de los últimos salarios recibidos), y desigualdades de género.

En respuesta a estos desafíos, la Comisión Bravo elaboró varias recomendaciones. Entre otras medidas, propusieron i) fortalecer y ampliar los subsidios públicos para los ingresos de pensiones, tanto en términos de cobertura (del 60\% al 80\%) como del monto de los ingresos (en un 20\%); ii) que no sólo los trabajadores, sino también los empleadores contribuyan a los ahorros de pensión (alrededor del 5\% del salario del trabajador); iii) que las personas que trabajan por cuenta propia contribuyan a sus pensiones mediante la promoción de incentivos financieros; iv) que una parte de las contribuciones de los nuevos empleadores se destine a un fondo compartido que beneficie a las personas con menores ingresos por pensiones, y v) reducir las brechas de género igualando la edad de jubilación de hombres y mujeres, así como eliminando el uso de las tasas de mortalidad por género para calcular la pensión mensual individual. ${ }^{3}$

Adicionalmente, otra de las iniciativas que tanto la mencionada Comisión como otras autoridades políticas han promovido para amortiguar los efectos financieros asociados al envejecimiento de la población consiste en incrementar la edad legal de jubilación y que las personas, así extiendan su vida laboral. Tener carreras laborales más largas e incrementar gradualmente la edad de jubilación permitiría a las personas acceder a una remuneración por mayor tiempo (la mayoría de las veces de un monto superior a una potencial pensión), y, además, aumentar el fondo acumulado de cotizaciones previsionales. Por otra parte, el hecho de que para solventar financieramente

2 Conocida como "Comisión Bravo" en atención a su presidente, el economista David Bravo.

3 Comisión Asesora Presidencial sobre el Sistema de Pensiones, Informe Final, Santiago de Chile, Gobierno de Chile, 2015. 
la jubilación se acuda a iniciativas vinculadas al mundo del trabajo evidencia la estrecha relación que existe entre el mercado laboral y los sistemas de pensiones.

En la misma línea, recientemente el actual presidente Sebastián Piñera anunció un proyecto de reforma al sistema previsional, que fue ingresado al Congreso el 6 de noviembre de 2018 (Mensaje 171-366, del 29 de octubre de 2018). La reforma reconoce que el sistema actual entrega pensiones de jubilación por debajo de lo esperado, y que uno de los factores que inciden en dicho resultado es el aumento de las expectativas de vida y la necesidad de que las pensiones financien una vejez más extensa. Una de las principales modificaciones propuestas consiste en la promoción de incentivos para postergar la jubilación definitiva del mercado laboral, tales como la posibilidad de realizar retiros parciales de dinero, la cuenta de ahorro previsional, después de la edad legal de jubilación, así como rebajas o eliminación de comisiones de las administradoras de fondos de pensión (AFP) para quienes continúen trabajando una vez cumplida la edad legal para pensionarse.

Independientemente de los argumentos a favor y en contra de políticas públicas que busquen postergar la edad de jubilación, la literatura internacional ha demostrado que este tipo de medidas poseen un efecto altamente positivo en la situación económica de las personas en la vejez. Sin embargo, aunque ello es empíricamente irrefutable, creemos que el proceso de reflexión de política pública en torno a la postergación de la edad de jubilación y a la extensión de las trayectorias laborales debe considerar no solamente los beneficios financieros de continuar con la actividad laboral, sino otros aspectos del mundo del trabajo (por ejemplo, las condiciones y garantías laborales mínimas para las personas mayores), así como otros dominios de vida de las personas mayores, tales como la salud, las diferencias de género, y los cambios culturales y tecnológicos propios del envejecimiento poblacional.

Basados en evidencia empírica nacional e internacional, en el presente artículo planteamos una discusión sobre diferentes dimensiones involucradas en las políticas que buscan incentivar el empleo en personas mayores y aplazar la transición a la jubilación. En concreto, en primer lugar discutimos la actual participación laboral de hombres y mujeres mayores, sus condiciones laborales, y cuáles han sido las trayectorias laborales y de cotización de los individuos que hoy están próximos a la transición a la jubilación. En segundo término, nos referimos a la manera en que se ha abordado la extensión 
de las carreras laborales y el incremento de la edad legal de jubilación en diferentes países. En tecer lugar, discutimos los principales desafíos de salud que actualmente enfrentan las personas mayores en Chile. En cuarto lugar, nos referimos al contexto cultural vinculado al envejecimiento poblacional en este país. Luego de esto, en la conclusión, resumimos los aspectos discutidos y proponemos posibles orientaciones que, desde nuestro punto de vista, debería tener una política pública que busque incentivar la postergación de la jubilación en Chile.

\section{CONTEXTO LABORAL Y DE PENSIONES DE LAS PERSONAS MAYORES EN CHILE}

\section{Participación de personas mayores en el mercado laboral}

Los datos de la Encuesta de Caracterización Socioeconómica Nacional (Casen) en Chile muestran que a partir de 2006, una proporción cada vez mayor de personas mayores participan en la fuerza laboral (tabla 1). Concretamente, el $72.6 \%$ de los hombres en el grupo de 60 a 64 años trabajaba en 2006 , mientras que en 2017 , el $82.5 \%$ de ellos se encontraba en el mercado laboral.

Las mujeres han enfrentado una tendencia similar, aunque las tasas de participación en la fuerza laboral son menores. Ello podría explicarse porque la incorporación de las mujeres al mercado de trabajo en las últimas décadas en Chile ha sido más tardía y, en general, de forma más precaria, atendidas las condiciones laborales que se ofrecen. Así, tradicionalmente, las mujeres han ocupado sectores específicos de producción, como es el de servicios, y con remuneraciones por debajo de las ofrecidas para los hombres. Otro factor relevante es la asociación de la mujer con las tareas relativas al cuidado familiar al traspasarles los costos laborales de la maternidad, lo cual ha tenido un impacto negativo en su inserción al mercado de trabajo. 
Esta revista forma parte del acervo de la Biblioteca Jurídica Virtual del Instituto de Investigaciones Jurídicas de la UNAM http://www.juridicas.unam.mx/

TABLA 1

TASA DE PARTICIPACIÓN LABORAL (\%) POR GÉNERO Y GRUPO ETARIO4

\begin{tabular}{c|c|cccccc}
\hline & $\begin{array}{c}\text { Grupo } \\
\text { etario }\end{array}$ & 2006 & 2009 & 2011 & 2013 & 2015 & 2017 \\
\hline \multirow{2}{*}{ Hombre } & $60-64$ & 72.6 & 73.7 & 74.7 & 78.1 & 80.6 & 82.5 \\
& $65+$ & 29.4 & 25.2 & 26.0 & 29.4 & 36.1 & 38.0 \\
\hline \multirow{2}{*}{ Mujeres } & $60-64$ & 29.3 & 27.5 & 29.1 & 34.6 & 37.6 & 39.9 \\
& $65+$ & 9.4 & 7.4 & 7.9 & 9.5 & 13.3 & 14.8 \\
\hline
\end{tabular}

Como se observa en la tabla 2, en comparación con el resto de los países de la OECD, la participación laboral de individuos mayores de 65 años en Chile es claramente mayor. ${ }^{5}$

TABLA 2

TASA DE PARTICIPACIÓN LABORAL (\%) DE PERSONAS

MAYORES DE 65 AÑOS 6

\begin{tabular}{c|cccccc}
\hline & 1996 & 2000 & 2004 & 2008 & 2012 & 2016 \\
\hline Chile & 18.7 & 17.3 & 17.0 & 19.8 & 23.0 & 24.3 \\
$\begin{array}{c}\text { OECD } \\
\text { Countries }\end{array}$ & 9.7 & 9.0 & 11.0 & 12.2 & 13.2 & 14.4 \\
\hline
\end{tabular}

Una explicación plausible para la alta participación laboral de las personas mayores en Chile corresponde a las necesidades financieras que enfrentan muchos trabajadores sin ahorros previsionales adecuados para su vida posterior, lo cual los obliga a trabajar más allá de la edad legal de jubilación. ${ }^{7}$ De

4 Casen, Adultos mayores (síntesis de resultados), Santiago de Chile, Ministerio de Desarrollo Social, 2017.

5 OECD, Pensions at a Glance 2015: OECD and G20 indicators, Paris, OECD Publishing, 2015; OECD, Labour force participation rate (indicator), 2017, doi: 10.1787/8a801325-en.

6 Idem.

7 Así también lo ha señalado la OIT, al indicar que Chile es uno de los países de la OECD donde los ingresos de las persones mayores provienen de sus trabajos, y no de una prestación de seguridad social, ILO, Social Protection for older Persons: Key Policy Trends and Statistics, 2014, p. 2. 
acuerdo con la regulación vigente, las mujeres pueden optar a la pensión de jubilación a partir de los sesenta años, mientas que los hombres a los 65 años. ${ }^{8}$ Sin embargo, el cumplir con el requisito de edad no significa que, necesariamente, las personas deban acogerse a una pensión y por lo tanto dejar de trabajar. Cumplir la edad de jubilación no constituye una causa legal para poner término al contrato de trabajo, por lo cual, siempre será voluntario continuar trabajando o no. Tampoco pone término al contrato el recibir una pensión de jubilación, ya que ésta no es incompatible con la remuneración que perciba el trabajador con motivo de su contrato, sin perjuicio de que en dicho caso se termina la obligación de cotizar para el sistema previsional.

Dos estudios recientes ${ }^{9}$ indican que mientras las necesidades financieras son el motivo más importante para prolongar la vida laboral, los problemas de salud y otros tipos de deberes son las razones principales para salir del mercado laboral en edades tempranas.

Una de las principales maneras que los adultos mayores en Chile usan para mantenerse activos en el mercado laboral es el trabajo por cuenta propia. ${ }^{10}$ En concreto, la Encuesta Nacional de Empleo de 2017 muestra que el 48.6\% de los adultos mayores activos trabajan en esta modalidad laboral. Sin embargo, como señala la literatura especializada, la mayoría de los trabajos por cuenta propia entre adultos mayores ocurren en el sector informal $53.7 \%$, específicamente, en Chile $)^{11}$ lo cual a menudo conduce a condiciones de trabajo precarias y menores ingresos: de acuerdo a registros oficinales del Instituto Nacional de Estadísticas, mientras la mediana de salarios para todos/as los/as trabajadores/as por cuenta propia en 2017 fue igual 201.177 (295,20 dólares), esta medida para trabajadores dependientes fue de 411.407 (603,68 dólares). ${ }^{12}$

8 La legislación vigente permite también la pensión anticipada, en caso de que se cumplan determinados requisitos de ahorro o de condiciones particulares de trabajo, artículos 68 y 68 bis del D. L. 3500.

9 Centro UC Políticas Públicas, Adultos mayores: un activo para Chile, Santiago de Chile, Pontificia Universidad Católica de Chile, 2017; CEVE UC, op. cit.

10 CEVE UC, op. cit.

11 Huenchuan, Sandra et al., Protección y participación en la vejez: escenarios futuros y políticas públicas para enfrentar el envejecimiento en Chile, Santiago de Chile, CEPAL, 2007; Vives, Alejandra et al., "Envejecimiento y trabajo en Chile: propuesta para el monitoreo de la salud laboural", Propuestas para Chile (Concurso Políticas Públicas), Santiago de Chile, Pontificia Universidad Católica de Chile, 2016; Instituto Nacional de Estadísticas, Encuesta Nacional de Empleo, 2017.

12 En adelante, se indican los montos en pesos chilenos (\$) y dólares americanos (USD), considerando un valor del dólar de referencia, de $\$ 681,5$. 


\section{Condiciones laborales de personas mayores}

Un indicador para evaluar las condiciones de trabajo de las personas mayores es el ingreso promedio. Los estudios muestran que en Chile el ingreso promedio de los trabajadores mayores de 65 años corresponde al 67\% del ingreso promedio del resto de la población más joven, mientras que en los países de la OECD esta cifra asciende al 86.2\%. ${ }^{13}$ Concretamente, según datos oficiales, la mediana de los ingresos laborales de las personas mayores de 65 años es de \$279.759 (USD 410,50), y existe una brecha importante entre hombres ( $\$ 320.000$ - USD 469,55$)$ y mujeres ( $\$ 150.883$ - USD 221,40).

Esta situación encuentra su explicación en la propia legislación laboral vigente, que establece una distinción respecto a la remuneración exigible para las personas mayores. Así, al fijarse el monto del ingreso mínimo mensual para todos los trabajadores que cumplan con una jornada ordinaria de trabajo, se señala que las personas mayores de 65 años tienen derecho al $75 \%$ de dicho monto que se establece por ley cada año. ${ }^{14}$ Por ejemplo, para 2018 el monto del ingreso mínimo mensual para estos trabajadores fue sólo de $\$ 214.99915$ (USD 315,48). La misma distinción se establece para los trabajadores que sean menores de dieciocho años, y el argumento que se ha utilizado para justificar que estos dos grupos etarios puedan obtener una remuneración inferior ha sido la menor productividad que supuestamente los caracteriza.

Claramente se trata de un criterio arbitrario y discriminador, que no se condice con las cifras indicadas oficiales, que muestran que una gran proporción de personas mayores de 65 años continúan trabajando, ${ }^{16}$ ni tampoco con los instrumentos internacionales relativos a estas materias. ${ }^{17}$ Además, como se ha señalado, uno de los motivos por los cuales las personas mayores siguen

13 Comisión Asesora Presidencial sobre el Sistema de Pensiones, Informe final, Santiago de Chile, Gobierno de Chile, 2015; OECD. Pensions at a Glance 2013: OECD and G20 Indicators, Paris, OECD Publishing, 2013.

14 Esta restricción aparece por primera vez en la Ley 19.307/1994, pero en la historia de la ley nunca se han explicado los motivos para esta diferenciación.

15 Ley 21.112, del 24 de septiembre de 2018, que fija el ingreso mínimo mensual desde el 1o. de septiembre de 2018 .

16 Además, esta diferenciación constituiría una vulneración al derecho fundamental a la igualdad. Véase, Rojas, Irene, Derecho del trabajo, Derecho individual del trabajo, Santiago, Chile, Thomson Reuters, 2015, p. 271.

17 La Recomendación 202 de la OIT (2012), sobre los pisos de protección social establece que constituye una de las garantías básicas de seguridad social el otorgar un ingreso para las personas de edad "por lo menos equivalente a un nivel mínimo definido en el plano nacional". 
trabajando cumplida la edad de jubilación es que sus ingresos son insuficientes, obligándolos a mantenerse laboralmente activos para aumentar sus ahorros previsionales, lo cual no se cumpliría si precisamente en esta última etapa laboral su remuneración es inferior a la que percibían en etapas previas de la vida.

El acceso a la capacitación y al perfeccionamiento laboral es otro indicador de las condiciones de trabajo en personas mayores. En un estudio reciente, el 20.8\% de los trabajadores mayores en Chile afirmó que les gustaría continuar aprendiendo nuevas competencias relacionadas con el trabajo. ${ }^{18}$ Sin embargo, otro estudio indicó que sólo el 4\% de los trabajadores de 60 a 74 años ha estado en una sesión de capacitación laboral de ocho horas (o más) durante el último año, ${ }^{19}$ lo que da cuenta de las bajas posibilidades de formación que existen en el mercado laboral para las personas mayores. Si este escenario continúa estructurado de este modo, los trabajadores mayores tendrán menos posibilidades de mantenerse en sus puestos de trabajo, o no serán considerados para ocupar nuevos cargos. La capacitación en este sentido es crucial para mantener vigentes las habilidades ocupacionales y, de este modo, permanecer vigentes en los puestos de trabajos o, eventualmente, optar a otros mejores. De lo contrario, los trabajadores mayores serán relegados a ocupaciones cada vez menos desafiantes, las que usualmente presentan condiciones laborales más precarias.

Otra forma de medir las condiciones de trabajo entre las personas mayores es a través de la evaluación de los riesgos para la salud en sus lugares de trabajo. Vives y otros autores ${ }^{20}$ evaluaron la exposición a riesgos laborales en cuatro dominios diferentes: higiene, ergonomía, seguridad y bienestar psicosocial. Entre otros resultados, encontraron que el 20\% de los individuos mayores de setenta años está empleado en trabajos que los obligan a estar expuestos a altas temperaturas y radiación solar (para los hombres, esta cifra aumenta a 37\%). Los riesgos ergonómicos más comunes para los trabajadores mayores son las posiciones difíciles, las tareas repetitivas y el trabajo de pie. Con respecto a los riesgos psicosociales en el trabajo, el 20\% de las personas mayores cree que el tiempo asignado para el trabajo es insuficiente para reali-

18 Programa Adulto Mayor UC, Chile y sus mayores. Resultados Tercera Encuesta Nacional Calidad de Vida en la Vejer, Santiago de Chile, Pontificia Universidad Católica de Chile y Caja Los Andes, 2013.

19 Centro UC, Políticas Públicas, Adultos mayores: un activo para Chile, Santiago de Chile, Pontificia Universidad Católica de Chile, 2017.

20 Vives, Alejandra et al., op. cit. 
zar sus tareas adecuadamente, el 50\% cree que sus trabajos implican una concentración excesiva, y el 45\% declara que sus trabajos son muy agotadores.

Por lo tanto, es razonable concluir que a pesar de los válidos motivos que tienen las personas mayores para mantenerse laboralmente activos, una vez cumplida la edad de jubilación, las condiciones que ofrece actualmente el mercado de trabajo en Chile no son las más apropiadas ni les aseguran garantías mínimas.

\section{Trayectorias laborales y de cotización en Chile}

Luego de 35 años de su introducción, el sistema de pensiones de capitalización individual ha comenzado a entregar, no exento de críticas, la primera ola de pensiones a personas que cotizaron, casi exclusivamente, en este nuevo esquema. ${ }^{21}$ Entre los múltiples cuestionamientos al sistema, uno de los que más destaca se refiere a las bajas pensiones que reciben los jubilados. El estudio "Desiguales" del Programa de las Naciones Unidas para el Desarrollo (PNUD) en Chile, de 2017 señala que el $72.5 \%$ de los jubilados reciben una pensión "bajo el mínimo" (lo que se define como inferior al 70\% del salario mínimo), vale decir menos de $\$ 193.200$ (USD 283,49). Por otra parte, respecto a la tasa de remplazo bruta en Chile, las cifras de la OECD indican que para hombres esta tasa es de $33.5 \%$, mientras que para las mujeres es $30.3 \% .^{22}$ En este punto cabe destacar que los responsables políticos y administrativos del nuevo sistema de capitalización individual estimaron que la tasa de reemplazo al final de la vida laboral sería alrededor de $70 \% .{ }^{23}$ El contexto actual indica que dicha estimación dista de la realidad.

21 La modificación al sistema de pensiones en Chile se realizó a través del Decreto Ley 3.500 de 1980, el cual estableció un sistema de capitalización individual obligatorio para todos quienes iniciaran su vida laboral a partir del 1o. de enero de 1983. Para quienes ya se encontraban trabajando, se dio la opción de que se cambiaran al nuevo modelo, o que pudieran continuar cotizando en las antiguas cajas previsionales que funcionaban bajo la lógica de un sistema de reparto, las que se fusionaron y quedaron bajo la administración del ex Instituto de Normalización Previsional (INP), actual Instituto de Previsión Social (IPS). Sobre las características del antiguo modelo previsional y la implementación del sistema de capitalización individual, véase Gumucio, Juan, "Una mirada al sistema de pensiones de administración privada de pensiones chileno al acercarse un cuarto de siglo de aplicación", Revista Latinoamericana de Derecho Social, núm. 2, enero-junio de 2006, pp. 175-199.

22 OECD, Gross pension replacement rates (indicator), 2018, doi: 10.1787/3d1afeb1-en.

23 Piñera, José, El cascabel al gato: la batalla por la reforma previsional, Santiago, Chile, Zig-Zag. 1991. 
Por otra parte, los responsables de este modelo de pensiones en Chile argumentan que los bajos montos de pensiones y las bajas tasas de remplazo son de exclusiva responsabilidad de cada trabajador por tener lagunas de cotización; esto es, haber pasado por periodos extensos de tiempo sin haber cotizado en sus fondos de pensiones, lo cual reduciría notoriamente la tasa de reemplazo, y en consecuencia, los ingresos durante la jubilación.

Sin embargo, es posible advertir otros factores que también inciden en que las pensiones sean insuficientes. En primer lugar, los bajos salarios durante la vida laboral de la gran mayoría de las personas es, ciertamente, un factor determinante, por cuanto a menor remuneración será también menor la cantidad de ahorros previsionales acumulados. En Chile, de acuerdo con los últimos datos oficiales, la mediana de ingresos es $\$ 379.000$ (USD 556,13). ${ }^{24}$ Otro factor corresponde al incumplimiento del pago de cotizaciones previsionales a las Administradores de Fondos de Pensiones (AFP) por parte de los empleadores, quienes están legalmente obligados a ello; sin embargo, en muchas ocasiones no lo cumplen, generando lagunas previsionales en sus trabajadores. Un tercer factor refiere que la administración de los ahorros previsionales realizada por las AFP está sujeta a las variaciones, muchas veces impredecibles del mercado financiero; en consecuencia, en algunos casos existen rentabilidades negativas de los ahorros previsionales de los cotizantes, lo cual tiene efectos directos en los ingresos que dispondrán durante la jubilación. A esto se agrega la generalizada falta de información y de transparencia de las inversiones financieras de las AFP, lo que incide en que los trabajadores desconozcan cómo funciona este sistema. ${ }^{25}$

Un cuarto y último factor, altamente asociado a las bajas pensiones, refiere a las condiciones del mercado laboral. ${ }^{26}$ En concreto, las estimaciones de los responsables de este sistema de pensiones sobre la tasa de remplazo en torno al $70 \%$ se basaban en el fuerte supuesto de que la mayoría de la población enfrentaría un "ciclo laboral convencional", el cual asume que las personas

24 Instituto Nacional de Estadísticas. Encuesta suplementaria de ingresos 2017, Santiago, Chile, Instituto Nacional de Estadísticas, 2018.

25 "La educación previsional juega un rol trascendental en el funcionamiento del sistema, en el entendido que sólo la información profunda permite al afiliado adoptar las decisiones que pueden llevarlo a un resultado exitoso". Véase en Lanata, Gabriela, Manual de legislación previsional, Santiago, Chile, Thomson-Reuters, 2015, p. 80.

26 Con relación a esta materia, resulta interesante revisar el informe de la Comisión Marcel (2006), que fue mandatada por la presidenta Bachelet durante su primer periodo de gobierno, con el propósito de realizar un diagnóstico de la situación previsional en Chile, y que se tomó como base para la Reforma Previsional de 2008 a través de la ley núm. 20.255. 
hasta alrededor de los vienticinco años adquieren competencias y habilidades educativas (técnicas o profesionales), para luego trabajar continuamente, sin interrupciones, en empleos a jornada completa (es decir, 45 horas a la semana), y, además, contribuyendo regularmente en sus fondos de pensión hasta la edad legal de jubilación, edad a partir de la cual transitarían hacia la inactividad laboral definitiva.

Los resultados de una reciente investigación ${ }^{27}$ evidencian que gran parte de las personas que iniciaron sus carreras laborales simultáneamente a la introducción del nuevo sistema de pensiones (vale decir, a partir de 1980) han estado alejadas del modelo del "ciclo laboral convencional". En concreto, los análisis indican que sólo en torno al 45\% de los individuos tuvo una trayectoria laboral consistente con lo esperado por los responsables de este esquema de pensiones; vale decir, una trayectoria caracterizada por personas que gran parte de su vida trabajan ininterrumpidamente como empleados, a tiempo completo, y cotizando continuamente a sus fondos de pensión. El resto de la población estudiada posee trayectorias caracterizadas por la ausencia permanente del mercado laboral (en torno al 31\% de la población), o por trayectorias en las que se presentan periodos de empleo (a cuenta propia o como dependientes), pero sin contribuciones a las cuentas individuales de ahorro previsional. Esto evidencia que el mercado laboral no se ha desarrollado en los términos proyectados para el éxito del sistema de capitalización individual.

Para entender por qué ocurre esta disociación entre el desarrollo del mercado laboral y del sistema de pensión en Chile, resulta importante entender el cambio de modelo previsional que se produjo, simultáneamente a un periodo de profundas reformas neoliberales, entre las que destacan las relaciones contractuales de trabajo. Específicamente, a través del conocido como "Plan Laboral" (Decreto Ley 2.200 de 1978) se introdujeron modificaciones que buscaban flexibilizar el sistema de relaciones individuales de trabajo, estableciendo facilidades para la contratación temporal, jornadas de trabajo extensas, bajas remuneraciones y flexibilidad en el sistema de terminación del contrato de trabajo a través de la incorporación de causales de despido amplias, y la restricción a los montos de las indemnizaciones por este motivo. Asimismo, es relevante mencionar que el sistema de pensiones en Chile exige la cotización obligatoria únicamente para trabajadores dependientes que se encuen-

27 Madero-Cabib, Ignacio, "Regímenes de pensiones con bases laborales precarias: Lecciones de un estudio longitudinal en Chile". Presentación en seminario Edad, trabajo y pensiones. Viejos y nuevos retos, Santiago, Flacso Chile y Centro de Estudios de Derecho del Trabajo y Seguridad Social de la Universidad de Talca, 2018 (inédito). 
tren sujetos a un contrato de trabajo, dejando por lo tanto fuera de su ámbito de aplicación a quienes se desempeñen en un mercado informal de trabajo o de manera independiente. ${ }^{28}$

Este escenario nos invita a reflexionar la relación entre trayectorias laborales y pensiones en dos sentidos específicos. En primer lugar, consideramos que los responsables políticos y administrativos de este sistema de pensiones deben integrar al análisis de las determinantes de la inequidad económica en la vejez el factor de la alta heterogeneidad de las trayectorias laborales en Chile (heterogeneidad que muy posiblemente aumentará en las próximas décadas, considerando la creciente flexibilidad en el mercado del trabajo). En segundo término, hacemos un llamado a los mismos responsables a fortalecer el componente no contributivo de las pensiones; esto es, crear mecanismos que aseguren bienestar financiero para todos los adultos mayores, independientemente de la participación laboral que hayan tenido durante el curso de vida.

\section{LA TENDENCIA HACIA LA EXTENSIÓN DE LAS TRAYECTORIAS LABORALES EN DIFERENTES PAÍSES}

A partir de la década del 2000 el escenario global de envejecimiento de la población, así como la inestabilidad financiera de los fondos de pensiones públicos y privados, fomentaron en diferentes países la necesidad de desincentivar la jubilación anticipada y, por el contrario, promover medidas institucionales para fomentar la jubilación tardía. ${ }^{29}$ Las políticas públicas que incentivan la extensión de las carreras de los trabajadores mayores han sido apoyadas por diversos organismos internacionales: por ejemplo, 2012 fue declarado por la Unión Europea el año para el envejecimiento activo, y, en el mismo año, la OECD sugirió aumentar gradualmente las edades para acceder a una pensión estatal. ${ }^{30}$

28 En efecto, en los últimos años una de las problemáticas que se ha levantado respecto a la cobertura del sistema previsional es que para los trabajadores independientes la obligación de cotizar había sido voluntaria, lo que se reflejaba en muy bajas tasas de afiliación. Esta situación se modificó a través de la Ley 20.255/2008, que estableció la obligación gradual de cotización para estos trabajadores a partir de 2012, modificación que tampoco mostró resultados positivos, y que llevó a dictaminar la Ley 20.894/2016, que prorrogó esta obligación hasta el 2019.

29 Madero-Cabib, Ignacio y Fasang, Anette, "Gendered Work-Family Life Courses and Financial Well-Being in Retirement”, Advances in Life Course Research, 2016, vol. 27, pp. 43-60.

30 OECD, OECD Pensions Outlook, 2012, Paris, OECD Publishing, 2012. 
El hecho de que existan iniciativas de política pública que promuevan la extensión de las trayectorias laborales en diferentes países implica que las salidas tardías del mercado laboral no sólo dependen de factores individuales como la salud, el nivel educativo y las preferencias personales, sino también del contexto institucional. Sin embargo, no todos los países utilizan los mismos mecanismos o procedimientos para incentivar la salida del mercado laboral. Por el contrario, este tipo de procedimientos dependen fuertemente del tipo de "Estado de bienestar" presente en cada nación.

El Estado de bienestar de un país es definido como el conjunto de políticas públicas y medidas institucionales utilizadas por cada nación para reducir las desigualdades de clase social y de género creadas en la sociedad. ${ }^{31}$ El Estado de bienestar puede ser de tipo liberal, corporativista, socialdemócrata, 'mediterráneo', o alguna combinación entre las opciones anteriores. La diferencia entre uno y otro tipo depende (entre otros elementos) de cuán determinante es el rol del mercado y del Estado para superar las diferencias de clase y género: mientras que algunos países promueven mecanismos administrados por el mercado (Estados liberales), otros países promueven mecanismos conducidos por organismos públicos (Estados socialdemócratas). ${ }^{32}$

En Estados de bienestar liberales, tales como el Reino Unido, Estados Unidos, Suiza, Canadá o Chile ${ }^{33}$ se ha observado que las personas extienden sus trayectorias laborales inducidos por la necesidad de tener un ingreso económico superior a la pensión entregada por esquemas previsionales, basados única o parcialmente en el pilar de capitalización individual (que obliga a las personas a tener altos ahorros para optar a una pensión adecuada para en-

31 "Por consiguiente, puede asegurar que el Estado del bienestar ha llegado a ser un elemento constituyente de la estructura política y social de las sociedades desarrolladas, en parte debido al crecimiento producido de la población que depende de las prestaciones sociales públicas (fundamentalmente trabajadores, pensionistas y empleados estatales)", Ochando, Carlos, ElEstado del bienestar: objetivos, modelos y teorías explicativas, Madrid, Ediciones Académicas, 2009 , p. 48.

32 Esping-Andersen, Gosta, Three Worlds of Welfare Capitalism, Princeton, NJ, Princeton University Press. 1990; Korpi, Walter, y Palme, Joakim, “The Paradox of Redistribution and Strategies of Equality: Welfare State Institutions, Inequality, and Poverty in the Western Countries", American Sociological Review, 1998, vol. 63(5), pp. 661-687.

33 "El Estado ha visto de hecho su participación limitada al rol de ente regulador, los agentes privados se han convertido en un elemento importante, incluso preponderante, en la gestión de la protección". Véase Arellano, Pablo, Universalismo e individualismo en el derecho chileno de pensiones, Santiago, Librotecnia, 2012, p. 36. 
frentar los riesgos financieros y de salud asociados a la vejez). ${ }^{34}$ Por otra parte, en países socialdemócratas, tales como Suecia, Dinamarca o Noruega, aunque también existen altos incentivos para mantenerse activo en el mercado laboral hasta edades tardías, a diferencia de los países liberales, esto es inducido mediante generosos beneficios financieros en las pensiones para toda la población, y, además, por políticas de capacitación laboral, que permiten a los trabajadores adaptarse a las condiciones actuales de flexibilidad laboral.

En síntesis, aunque en diferentes países la reacción al envejecimiento de la población y a la amenaza de la insostenibilidad financiera de los sistemas de pensiones ha sido eliminar cualquier tipo de incentivo hacia la jubilación anticipada, y por el contrario promover trayectorias laborales prolongadas, lo cierto es que los procedimientos para realizar esto varían entre diferentes regímenes de Estados de bienestar.

Nuestro país no ha estado ajeno a esta discusión. La preocupación por los problemas que presenta el sistema previsional ha aumentado a medida que más trabajadores deben jubilarse bajo el modelo de capitalización individual, sin haber podido ahorrar lo suficiente y con expectativas de vida más largas. Tanto los actores políticos como sociales han manifestado la necesidad de revisar las principales carencias del sistema de pensiones, para asegurar que las personas mayores puedan acceder a mejores pensiones. Dentro de este contexto, la necesidad o no de aplazar la edad de jubilación ha sido uno de los puntos más discutidos. Como ya se ha señalado, durante el primer gobierno de la presidenta Michelle Bachelet se creó la Comisión Marcel (2006), que aunque no se pronunció sobre la necesidad de aplazar la edad de jubilación, sí propuso eliminar la distinción de diferentes edades de jubilación entre hombres y mujeres, indicando que era uno de los factores que contribuían a la falta de equidad de género respecto de las pensiones de jubilación. La ley de reforma previsional 20.255 recogió estas ideas, al establecer como requisito para obtener prestaciones solidarias tener 65 años tanto para hombres como para mujeres. ${ }^{35}$

34 Buchholz, S. et al., "Globalization, Accelerating Social Change and Late Careers: A Theoretical Framework", 2006, en Blossfeld, H. P. et al., Globalization, Uncertainty and Late Careers in Society, Londres y Nueva York, Routledge, pp. 1-23.

35 Si bien la reforma previsional de 2008 no realizó modificaciones profundas al sistema previsional, manteniendo el esquema de capitalización individual obligatorio, sí incluyó diversas medidas para mejorar la situación de las personas que por diversas razones quedaban excluidas del sistema, y también para aquellos que habiendo cotizado regularmente sus pensiones no alcanzaban un monto mínimo definido. De esta manera, uno de los mayores aportes de esta reforma fue la inclusión de un "pilar solidario", que entrega prestaciones no contributivas. 
Por el contrario, el informe de la Comisión Bravo ${ }^{36}$ sí entregó propuestas sobre esta materia, indicando que debido al aumento de las expectativas de vida la incorporación de las personas mayores al mercado laboral resulta necesaria, pero acompañada de buenas condiciones laborales. ${ }^{37}$ De esta manera, el informe sugiere múltiples propuestas para extender las trayectorias laborales, tales como subsidios de empleo para las personas mayores, así como incrementos en los subsidios de pensiones para aquellos que posterguen la edad de jubilación. Sin embargo, las propuestas de este informe no se vieron materializadas en modificaciones legales.

Por otra parte, la Comisión Asesora de Pensiones de la Confederación de la Producción y el Comercio (CPC) realizó un informe en 2017, en el cual propuso igualar la edad de jubilación entre hombres y mujeres, retrasando la edad de jubilación para las mujeres, para luego aumentar la edad para ambos a 67 años. ${ }^{38}$

Finalmente, el mencionado proyecto de modificación al sistema previsional del presidente Sebastián Piñera propone extender la edad de jubilación de forma voluntaria a través de dos incentivos específicos. En primer lugar, promueve la posibilidad de realizar retiros de la cuenta individual de pensiones por un monto igual al 50\% de la diferencia entre el monto acumulado en la cuenta individual al momento de la jubilación definitiva y el monto exigido por las AFP para financiar una pensión (calculado a la edad legal de jubilación). Respecto a este primer incentivo, consideramos que una de sus dificultades es que opera sobre el supuesto de que las personas tendrán una diferencia positiva de dinero al momento de querer acceder a este incentivo. En otras palabras, quienes al momento de cumplir la edad de jubilación no cuenten con fondos suficientes para financiar su pensión (es decir, tengan una diferencia negativa de dinero en el cálculo recién explicado), no podrán hacer uso de este incentivo. Por otra parte, llama la atención que el incentivo

36 Comisión Asesora Presidencial sobre el Sistema de Pensiones, op. cit.

37 "Los antecedentes recopilados para el diagnóstico muestran que muchos trabajadores enfrentan dificultades en el mercado laboral en los años previos a la edad de jubilación, y después, en caso de seguir trabajando. La solución a estos problemas de precariedad laboral deben situarse en el lugar donde estos se originan, precisamente en el mercado de trabajo, y no en el sistema previsional", Comisión Bravo, 2015, p. 123.

38 "Los cambios en la edad de jubilación deben venir acompañados de propuestas específicas e incentivos que permitan aumentar la participación laboral de adultos mayores que quieran y puedan trabajar, por ejemplo, permitiendo el trabajo durante algunas horas al día, a través de una mayor flexibilidad horaria y el teletrabajo, entre otros". Véase Informe Comisión Asesora de Pensiones de CPC, 2017, p. 36. 
consista en poder retirar parte de los fondos de pensión ahorrados a pesar de continuar trabajando, pues esto supone que el mejoramiento de la situación financiera de cada persona mayor no se produce necesariamente por la extensión de la actividad laboral después de la edad legal de jubilación, sino exclusivamente gracias al acceso parcial a sus propios ahorros de pensión.

En segundo lugar, el proyecto del presidente Piñera propone un incentivo a través de la disminución o eliminación de las comisiones que exigen las AFP, ${ }^{39}$ específicamente para los trabajadores que una vez cumplida la edad de jubilación sigan cotizando para su pensión. Este mecanismo resulta más interesante desde nuestro punto de vista, ya que precisamente aborda un aspecto altamente criticado de las AFP: el costo de dichas comisiones. Por otra parte, este incentivo permitiría a las personas que sigan trabajando después de la edad legal de jubilación, tener un aumento directo de sus remuneraciones líquidas, pues pagarían menores o ninguna comisión a las AFP.

Sin perjuicio de que, posiblemente, este nuevo proyecto de reforma enfrentará diversas modificaciones durante el transcurso de su tramitación parlamentaria antes de su eventual aprobación, es válido preguntarse cuál será el efecto real de estas propuestas en el actual contexto del mercado laboral para las personas mayores en Chile. En efecto, presumimos que los incentivos a la extensión de las trayectorias laborales sin medidas simultáneas de mejoramiento de las condiciones laborales mínimas de las personas mayores generarán sólo un leve impacto positivo en sus condiciones financieras, pero podrían producir enormes perjuicios en otras dimensiones de la vida, como son la salud y el bienestar personal de este grupo social.

\section{LA DIMENSIÓN DE SALUD DE LAS PERSONAS \\ MAYORES EN CHILE}

El aplazamiento de la edad de jubilación y la extensión de las trayectorias laborales tienen como supuesto fundamental, que la mayoría de las personas mayores tendrán condiciones de salud adecuadas para seguir activas en el mercado del trabajo. En esta sección discutimos algunas de las prioridades de salud para las personas mayores en este país: la discapacidad, la dependencia, y las labores de cuidado.

39 Cotizaciones cobradas, principalmente, por administrar los fondos de pensiones. 


\section{Discapacidad en la vejez}

La discapacidad en adultos mayores es un tema que ha sido correctamente estudiado en Chile. Un ejemplo ilustrativo de esto es el desarrollo del Estudio Nacional de Discapacidad que realiza el Ministerio de Desarrollo Social, con dos objetivos principales: i) proporcionar un diagnóstico detallado sobre las condiciones de vida de la población en condiciones de discapacidad, y ii) proponer políticas públicas adecuadas para abordar los problemas de esta población. Los resultados de la versión 2016 de este estudio indican, primero, que $38.3 \%$ de la población adulta mayor (30.3\% de hombres y $44.3 \%$ de mujeres) reporta tener una discapacidad $(20.8 \%$ en grados parciales y $11.6 \%$ en grados severos); en segundo lugar, sólo el 16.5\% de las personas mayores con discapacidad siguen activas en el mercado laboral (mientras que el $40.6 \%$ de las personas mayores sin discapacidad lo hacen); en tercer lugar, las personas mayores en situación de discapacidad que continúan trabajando ganan un salario promedio igual a $\$ 260.931$ (USD 382,88), mientras que para los trabajadores mayores sin discapacidades el salario promedio es $\$ 390.803$ (USD 573,45), y, finalmente, el $51 \%$ de las personas mayores en situación de discapacidad reportan ser dependientes, el $62.8 \%$ reporta una enfermedad crónica, y el $73.9 \%$ de las personas mayores en condición de discapacidad, y que además son dependientes, son atendidas por cuidadores informales (principalmente mujeres no remuneradas).

Con respecto a las causas y consecuencias de estar en condiciones de discapacidad, un reciente estudio ${ }^{40}$ analizó seis tipos de discapacidades (visuales, auditivas, del habla, físicas, cognitivas, y psiquiátricas), y descubrió que quienes tienen menos probabilidades de enfrentar tales discapacidades son aquellos que permanecen activos en el mercado laboral, tienen un ingreso más alto, y un mayor nivel educativo. Por otra parte, con relación a las consecuencias de la discapacidad, un estudio referido específicamente a las inequidades en el acceso a la salud muestra que independientemente del nivel socioeconómico de los individuos, quienes están en condiciones de discapacidad enfrentan mayores dificultades para acceder a servicios de salud, programar una visita al médico, y obtener diferentes tipos de medicamentos. ${ }^{41}$ Esta investigación se-

40 Zitko, Pedro y Cabieses, Báltica, "Socioeconomic Determinants of Disability in Chile", Disability and Health Journal, 2011, vol. 4(4), pp. 271-282.

41 Rotarou, Elena, y Sakellariou, Dikaios, "Inequalities in Access to Health Care for People with Disabilities in Chile: the Limits of Universal Health Coverage", Critical Public Health, 2017, vol. 27(5), pp. 604-616. 
ñala, además, que estas inequidades son aún más evidentes para las personas en situación de discapacidad que se atienden en el sistema público de salud.

\section{Dependencia, demencia y cuidados en la vejez.}

Con relación a la dependencia, un reciente estudio analizó los factores de riesgo de esta condición entre un grupo de adultos mayores en Chile. ${ }^{42}$ Utilizando datos de la Encuesta Nacional de la Dependencia en las Personas Mayores, los autores identificaron que además del conocido efecto negativo del envejecimiento sobre la dependencia existen algunos factores psicosociales que retrasan el inicio de la dependencia en la vejez, tales como un mayor nivel educativo, mantener un contacto constante con la familia o el núcleo de personas cercanas, además de realizar actividades extraprogramáticas, como leer o hacer ejercicio. Esta investigación, además analizó las condiciones de cuidado de las personas mayores dependientes, cuidado que a menudo es proporcionado informalmente por mujeres de su familia. Los autores concluyen que en algunas ocasiones el cuidado de personas mayores dependientes posee efectos negativos tanto en el bienestar subjetivo de las cuidadoras (generando estrés, depresión y fatiga física) como en su bienestar financiero (ausencia de ingresos, además de interrupciones en las contribuciones previsionales por tratarse de un trabajo informal).

En cuanto a las políticas públicas sobre dependencia en personas mayores en Chile, hasta el momento se han desarrollado tres programas claves: i) "Evaluación Funcional del Adulto Mayor" (1995), el cual se creó para identificar a las personas mayores en riesgo de dependencia mediante la evaluación de su rendimiento funcional y cognitivo; ii) "Más Adultos Mayores Autovalentes" (2015), cuyo objetivo es mantener funcionalmente activas a aquellas personas que han sido clasificadas en riesgo de enfrentar dependencia, y iii) "Chile Cuídeme" (2013), que ofrece apoyo psicológico a los/as cuidadores familiares de personas dependientes. ${ }^{43}$

Otras investigaciones sobre la dependencia entre adultos mayores han mostrado que esta condición es más frecuente entre grupos socioeconómicos

42 Cheix, Consuelo et al., "Risk Factors of Dependency of Chilean Older Adults and Consequences on Informal Caregiving”, América Latina Hoy, 2015, vol. 71, pp. 17-36.

43 Thumala, Daniela et al., "Aging and Health Policies in Chile: New Agendas for Research", Health Systems y Reform, 2017. 
bajos, ${ }^{44}$ y que la demencia (experimentada por el $7 \%$ de individuos mayores de sesenta años, $7.7 \%$ de mujeres y $5.9 \%$ hombres) se destaca como una determinante crucial de la dependencia. ${ }^{45}$ Este último estudio enfatiza, además, que a pesar del alto impacto de la demencia en el deterioro de la calidad vida de las personas mayores, esta condición no está clasificada como una de las enfermedades apoyadas financieramente por el Estado; por lo tanto, no tiene prioridad en los servicios de salud.

Por su parte, otra investigación reciente evaluó los costos económicos de la demencia en Chile. ${ }^{46}$ Utilizando datos primarios de una encuesta realizada a 330 personas mayores con demencia y atendidas por cuidadores informales, el estudio identifica tres dimensiones principales de los costos económicos asociados a la demencia: i) costos médicos directos (cuidados, tratamientos, medicamentos); ii) costos sociales directos (servicios sociales de cuidado), y iii) costos sociales indirectos (aquellos costos relacionados con el cuidado informal). Al contrario de lo que ocurre en países desarrollados, los costos indirectos en Chile representan el 75\% de los costos totales de la demencia, y está, inversamente relacionado con el estatus socioeconómico: en los hogares más pobres en los que los bajos ingresos no permiten acceder una atención de cuidado formal, los miembros de la familia (en su mayoría mujeres) no tienen otra opción que asumir el rol de cuidador. Los autores enfatizan, por lo tanto, que el estatus socioeconómico y el género son factores clave relacionados con el costo de la demencia en este país.

Por último, otro estudio ${ }^{47}$ destaca que el financiamiento público a servicios de cuidado para personas mayores, aunque se ha incrementado en los últimos años, continúa siendo muy insuficiente. Los autores argumentan que esto tiene consecuencias directas en diferentes dimensiones de estos servicios, tales como la disponibilidad, la accesibilidad, la equidad, la libertad de elección, la autonomía personal, la participación social, la exigibilidad de derechos, el acceso a información, y la rendición de cuentas.

44 Fuentes-García, Alejandra et al., "Socioeconomic Inequalities in the Onset and Progression of Disability in a Cohort of Older People in Santiago", Gaceta Sanitaria, Santiago, 2013, vol. 27(3), pp. 226-232.

45 Thumala, Daniela et al., op. cit.

46 Hojman, Daniel et al., "The Cost of Dementia in an Unequal Country: The case of Chile", PloS One. 2017, vol. 12(3).

47 Cunill-Grau, Nuria y Leyton, Cristian, "Una nueva mirada al enfoque de derechos. Aportes al debate a partir de los servicios de cuidado para personas mayores en Chile", Revista Latinoamericana de Derecho Social, vol. 27, 2018, pp. 23-57. 


\section{La relación entre trabajo y salud en la vejez.}

Los resultados de un proyecto de investigación que examinó el efecto de trayectorias laborales y del momento de jubilación en la salud de adultos mayores en Chile y otros once países del mundo ${ }^{48}$ indican, entre otros resultados, que las personas que han podido mantener trayectorias laborales continuas (sin interrupciones) en condiciones formales, y trabajando a tiempo completo, son más susceptibles de enfrentar mejores estados de salud física y cognitiva en la vejez. Por otra parte, los individuos que se retiran tardíamente del mercado del trabajo, así como los que se retiran de manera gradual (esto es, reduciendo paulatinamente el número de horas semanales destinadas al trabajo), son menos susceptibles de enfrentar accidentes cardiovasculares en la vejez.

Asimismo, esta investigación evidenció que la relación entre carreras laborales, jubilación y salud en la vejez depende no solamente de factores individuales (por ejemplo, tipo de trabajo, condiciones de formalidad laboral, horas de trabajo por semana, u otras características, como el género o el nivel educativo y socioeconómico de cada persona), sino que además está mediada por factores institucionales y políticas públicas que definen el Estado de bienestar en cada país. $\mathrm{Al}$ respecto, el estudio indica que los patrones laborales que derivan en una jubilación anticipada (antes de la edad legal de jubilación) tienen un impacto negativo en indicadores de salud, tales como enfermedades crónicas y escalas subjetivas de salud. Sin embargo, esta asociación es particularmente negativa en países que tienen Estados de bienestar de orientación liberal, donde los riesgos financieros y de salud asociados a la vejez son asumidos individualmente y no entre diferentes generaciones, como ocurre en países corporativistas o socialdemócratas.

\section{DIMENSIÓN CULTURAL DE LAS PERSONAS MAYORES EN CHILE}

Otro de los supuestos de la introducción de medidas institucionales que buscan aplazar la edad de jubilación y extender las carreras laborales de las

48 Madero-Cabib, Ignacio, Proyecto Fondecyt Postdoctorado 3160522, “The Impact of Employment Trajectories and Retirement Transition Timing on Older Adults' Health: a Longitudinal Study in 11 Countries", 2018. 
personas mayores, es que este tipo de iniciativas se desarrollen dentro un contexto cultural acorde. En concreto, el supuesto es, en primer lugar, que las personas mayores poseen la predisposición individual a continuar trabajando hasta edades tardías, y en segundo término, que el resto de la sociedad permitirá (y no discriminará) a las personas mayores que continúen ejerciendo un empleo. En esta sección discutimos tanto las percepciones de las personas mayores respecto al trabajo en edades tardías y al sistema de pensiones como los estereotipos que existen en torno a la vejez.

\section{Actitudes y opiniones sobre la jubilación y el sistema de pensiones}

Otra tarea realizada por la Comisión Bravo a cargo de la evaluación del sistema de pensiones en 2014 fue el desarrollo de una encuesta que examinó las actitudes y opiniones hacia la jubilación y el sistema de pensión de capitalización individual. Una de las preguntas de la encuesta (pregunta de respuesta múltiple) se refería a la forma en que los encuestados planeaban financiar (o financian actualmente) sus vidas en la vejez. La mayoría de ellos respondieron a través de una pensión individual de las AFP (51\%); a través de ahorros personales (32\%), y a través de subsidios estatales (25\%), mientras que el $14 \%$ de los encuestados respondió que no lo ha pensado todavía. Otra pregunta refirió si las personas estarían dispuestas a trabajar cinco años más, después de la edad de jubilación, para aumentar sus ahorros de pensión. Sólo el 29\% de los individuos estaban a favor, mientras que el $48 \%$ estaba en contra. Finalmente, el 71\% de las personas jubiladas declaró que sus ingresos de pensión no son suficientes para cubrir sus necesidades diarias.

Otro estudio de 2017 destacó que la mayoría de las personas mayores de 65 años tienen una evaluación negativa sobre el desempeño del sistema de pensiones actual en Chile. ${ }^{49}$ Sin embargo, los trabajadores de veinticinco a sesenta años de edad tienen una visión un poco más crítica de la institución de pensiones que los jubilados. Por ejemplo, mientras que el 55\% de los jubilados declara que su estado económico ha disminuido después de la jubilación, el 58\% de los trabajadores entre veinticinco y sesenta años cree que después de la jubilación su estado económico disminuirá. Asimismo, el 13\% de los jubilados declara que la situación económica mejoró después de la jubilación,

49 Browne, Magdalena y Valdés, Salvador, De la decepción de los pensionados al temor de los ocupados, Santiago de Chile, Centro CLAPES UC, 2017. 
mientras que sólo el 8\% de los trabajadores entre veinticinco y sesenta años de edad esperan que su situación económica mejore después de la jubilación.

\section{Discriminación contra las personas mayores (o la discriminación por edad)}

En Chile, los estereotipos negativos hacia la población mayor (o prejuicios viejistas) están relacionados con el aparente deterioro de su desempeño cognitivo, biológico y social..$^{50}$ Por ejemplo, el $72.9 \%$ de los chilenos piensa que los adultos mayores no pueden manejarse solos, ${ }^{51}$ aunque este tipo de prejuicio viejista existe, principalmente entre los jóvenes con educación superior. ${ }^{52}$ Otro estudio que examinó los estereotipos de los adultos mayores, específicamente dentro de las organizaciones de trabajo, concluye que las expectativas negativas de los trabajadores mayores a menudo se asocian con la disminución de sus habilidades cognitivas y físicas, lo que a su vez afecta su desempeño laboral..$^{53}$ Los autores de esta investigación afirman que en Chile todavía hay pocas medidas para prevenir el viejismo, no sólo dentro de las organizaciones de trabajo, sino en la sociedad en general. En otras palabras, el viejismo debería ser considerado seriamente como una expresión de discriminación contra un grupo vulnerable de la población. ${ }^{54}$

Desde nuestro punto de vista, la discriminación laboral de personas mayores y la falta de mecanismos eficaces para enfrentarla es un problema que debe analizarse en clave de derechos humanos, particularmente teniendo en cuenta la interpretación evolutiva de los derechos y el principio favor persona, considerando al efecto la edad como una categoría suceptible de distinción arbitraria, justificando la procedencia de exámenes más estrictos para determinar su compatibilidad con la Constitución y con las obligaciones internacionales del Estado en materia de derechos fundamentales. La preocupación

50 Abusleme, Maria Teresa y Guajardo, Gabriel, El maltrato hacia las personas mayores en la Región Metropolitana, Chile, Santiago de Chile, Senama-FACSO, Universidad de Chile, 2013; Thumala, Daniela et al., op. cit.

51 Thumala, Daniela et al., Inclusión y exclusión social de las personas mayores en Chile, Santiago de Chile, Ediciones Servicio Nacional del Adulto Mayor, Senama-FACSO, Universidad de Chile, 2015.

52 Arnold, Marcelo et al., "Young People's Images of Old Age in Chile: Exploratory research”, Educational Gerontology, 2008, vol. 34(2), pp. 105-123.

53 Nazar, Gabriela y Figueroa, Carolina, "Creencias estereotípicas sobre el desempeño laboral de trabajadores mayores en Chile”, Psicoperspectivas, 2015, vol. 14(1), pp. 114-125.

54 Thumala, Daniela et al., op. cit. 
por la discriminación por edad en el trabajo es una forma de discriminación recientemente reconocida, en múltiples países del mundo, incluido Chile. Tiene reconocimiento en diversos instrumentos internacionales, destacando el Convenio 111 OIT y la Convención Interamericana sobre la Protección de los Derechos Humanos de las Personas Mayores, de reciente vigencia. Por su parte, el reconocimiento normativo a nivel interno viene dado, fundamentalmente, por el artículo 19, numerales 2o. y 16 de la Constitución, y su desarrollo legal en el artículo 2o. del Código del Trabajo.

Una de las alternativas para mejorar el sistema de protección frente a discriminaciones por edad corresponde a políticas de empleo que promuevan la igualdad de trato por edad, ${ }^{55}$ incluyendo, eventualmente, medidas especiales de discriminación positiva ${ }^{56}$ para personas de edad avanzada. ${ }^{57} \mathrm{Al}$ respecto, cabe citar, especialmente, a la Convención Interamericana sobre la Protección de los Derechos Humanos de las Personas Mayores, que en su artículo 18 reconoce el derecho al trabajo. En lo pertinente establece que "los Estados Parte adoptarán las medidas legislativas, administrativas o de otra índole para promover el empleo formal de la persona mayor y regular las distintas formas de autoempleo y el empleo doméstico, con miras a prevenir abusos y garantizar una adecuada cobertura social y el reconocimiento del trabajo no remunerado". Asimismo, "los Estados Parte promoverán programas y medidas que faciliten una transición gradual a la jubilación, para lo cual podrán contar con la participación de las organizaciones representativas de empleadores y trabajadores y de otros organismos interesados". Promoverán también "políticas laborales dirigidas a propiciar que las condiciones, el ambiente de trabajo, horarios y la organización de las tareas sean adecuadas a las necesidades y

$55 \mathrm{Al}$ parecer, es necesario "demostrar cómo el mantenimiento de estos trabajadores en el empleo y la contratación puede formar parte integrante de la gestión empresarial competitiva y productiva y ser compatible con la misma". OIT, La igualdad en el trabajo: un objetivo que sigue pendiente de cumplirse. Informe global con arreglo al seguimiento de la Declaración de la OIT relativa a los principios y derechos fundamentales en el Trabajo, Conferencia Internacional del Trabajo, $100^{\circ}$ reunión, Ginebra, OIT, 2011, p. 73.

56 Arese, César, "Gran avance en derechos humanos laborales de las personas mayores", Estudios Latinoamericanos de Relaciones Laborales y Protección Social, Madrid, Cinca, 2016, vol. I, núm. 1, p. 28. Pueden revisarse algunas experiencias nacionales en esta línea, en OIT, 2011, op. cit., pp. 55 y 56 .

57 Respecto del sistema europeo y sistemas nacionales en dicho contexto geográfico hay nutrida literatura. Por todos, véase Calvo, Javier, "Los mayores en el nuevo derecho del empleo", en Galán, Agustín, Transiciones laborales y reformas del mercado de trabajo: su influencia sobre el empleo de jóvenes y mayores, Albacete, Bomarzo, 2015. 
características de la persona mayor", y "alentarán el diseño de programas para la capacitación y certificación de conocimiento y saberes para promover el acceso de la persona mayor a mercados laborales más inclusivos". 58

\section{DISCUSIÓN Y CONCLUSIÓN}

- Actualmente el sistema previsional en Chile se encuentra en crisis por cuanto las pensiones entregadas por el actual modelo de capitalización individual administrado por AFPs han demostrado ser insuficientes para la gran mayoría de los pensionados.

- El hecho de que las pensiones de vejez deban autofinanciarse a través del ahorro individual, y además, que ni los empleadores ni el Estado contribuyan a ese ahorro, implica que las pensiones dependen casi exclusivamente de la trayectoria laboral que haya desarrollado cada persona, y de las condiciones y garantías de que haya podido gozar durante su vida laboral.

- Atendidas las particularidades del sistema de relaciones de trabajo en Chile y de las características del modelo previsional, el nivel de ahorro, en general, no es suficiente para que los trabajadores logren reunir un monto que permita acceder a prestaciones adecuadas al cumplir la edad de jubilación. Ello implica que los trabajadores deben extender sus trayectorias laborales a fin de aumentar sus ahorros previsionales o para poder cubrir sus necesidades básicas, resultando entonces en un debate abierto y bifronte sobre la edad legal de jubiliación: sobre su determinación (debe o no aumentarse), y sobre si debe ser obligatoria y automática (causa de terminación del contrato de trabajo), o, voluntaria, dejando

58 En el ámbito europeo resulta de interés, en esta misma perspectiva, el Acuerdo Marco Autónomo sobre Envejecimiento Activo y Enfoque Intergeneracional. Se trata de un acuerdo marco suscrito el 8 de marzo de 2017 por los principales interlocutores empresariales y sindicales europeos, revestidos de amplia representatividad. Su propósito central es facilitar la participación activa de los trabajadores de más edad en el mercado laboral hasta la edad de jubilación y promover un enfoque intergeneracional. El acuerdo es buen indicador del estado actual de la discusión en Europa sobre el maltrato estructural de los adultos mayores en el ámbito laboral, y pone en relieve al diálogo social como mecanismo para avanzar en estas materias. Véase la traducción al castellano en Palomo, Rodrigo y Salazar, Fernanda, "Acuerdo marco autónomo sobre envejecimiento activo y enfoque intergeneracional en la Unión Europea", Revista Laboral Chilena (julio), Santiago de Chile, Ediciones Jurídicas Ltda, 2017, pp. 103-107. 
al trabajador la posibilidad de continuar trabajando, como actualmente ocurre en este país.

- Considerando el aumento dramático de las expectativas de vida, así como el aumento de trabajadores sujetos al sistema previsional de capitalización individual, en Chile, durante los últimos años se ha comenzado a enfrentar este contexto llevando a cabo diversos estudios desde el Estado y la academia, para tener rigurosos diagnósticos y sugerencias sobre cómo abordar los principales desafíos.

- Intentando abordar los problemas clave del sistema de pensiones en este país, la reciente propuesta de reforma al modelo previsional del presidente Sebastián Piñera propone incentivos para extender la edad de jubilación, con el propósito de aumentar los ahorros previsionales en esta última etapa laboral.

- A pesar de que esta reforma en principio tendría un efecto positivo directo en los montos de las pensiones, es posible anticipar que si las condiciones de trabajo no son adecuadas no necesariamente se configura como un incentivo positivo. En otros términos, si las remuneraciones laborales son bajas durante la vejez, el impacto de permanecer mayor tiempo en el mercado del trabajo sobre los montos de las pensiones no será el esperado.

- Lo importante de la reforma, sin embargo, es que permitirá discutir adecuadamente la necesidad de extender la edad de jubilación, así como su potencial impacto en dimensiones de salud, culturales y de género entre las personas mayores.

- Respecto a ello, consideramos que la extensión de las trayectorias laborales puede tener efectos positivos para las personas mayores en diversos aspectos de su vida, pero no necesariamente será un beneficio si no se consideran adecuadamente aspectos laborales y contractuales, el contexto cultural y las condiciones mínimas relativas a la salud de las personas mayores, asociadas a la introducción de este tipo de medidas institucionales.

- En consecuencia, la extensión de las trayectorias laborales y el aplazamiento de la edad legal de jubilación debe ir acompañada de garantías mínimas que permitan asegurar condiciones dignas de trabajo para las personas mayores. Sin perjuicio de los problemas propios del sistema previsional de capitalización individual, el problema de fondo se origina en las malas condiciones laborales, e impacta en las prestaciones de seguridad social. Un cambio efectivo supone, necesariamente, una revisión profunda a estas dos áreas. 


\section{REFERENCIAS}

Abusleme, María Teresa y GuajARdo, Gabriel, El maltrato hacia las personas mayores en la región metropolitana, Chile, Santiago de Chile, Senama-FACSOUiversidad de Chile, 2013.

Arellano, Pablo, Universalismo e individualismos en el derecho chileno de pensiones, Santiago de Chile, Librotecnia, 2012.

ARESE, César, "Gran avance en derechos humanos laborales de las personas mayores", Estudios Latinoamericanos de Relaciones Laborales y Protección Social, Madrid, Cinca, vol. I, núm. 1, 2016.

ARNOLD, Marcelo et al., "Young People's Images of Old Age in Chile", Exploratory Research. Educational Gerontology, 34(2), 2008.

Bravo, David y HugheS, E., Encuesta Calidad de Vida del Adulto Mayor e Impacto del Pilar Solidario, Santiago de Chile, Centro UC Encuestas y Estudios Longitudinales, 2018.

BROWNe, Magdalena y VALDÉs, Salvador, De la decepción de los pensionados al temor de los ocupados, Santiago de Chile, Centro CLAPES UC, 2017.

BuchHolz, S. et al., "Globalization, Accelerating Social Change and Late Careers: A Theoretical Framework", en BLOSSFELD, H. P. et al., Globalization, Uncertainty and Late Careers in Society, Londres y Nueva York, Routledge, 2006.

CALVO, Javier, "Los mayores en el nuevo derecho del empleo", en GALÁN, Agustín, Transiciones laborales y reformas del mercado de trabajo: su influencia sobre el empleo de jóvenes y mayores, Albacete, Bomarzo, 2015.

CASEN, Adultos Mayores (Sintesis de resultados), Santiago de Chile, Ministerio de Desarrollo Social, 2017.

Centro UC Políticas Públicas, Adultos mayores: un activo para Chile, Santiago de Chile, Pontificia Universidad Católica de Chile, 2017.

CEVE UC, Trabajo y personas mayores en Chile: lineamientos para una politica de inclusión laboural, Santia de Chile, Pontificia Universidad Católica de Chile, 2018.

CHEIX, Consuelo et al., "Risk Factors of Dependency of Chilean Older Adults and Consequences on Informal Caregiving", América Latina Hoy, 2015.

Comisión Asesora Presidencial sobre el Sistema de Pensiones (COMisión Bravo), Informe Final, Santiago de Chile, Gobierno de Chile, 2015. 
Comisión Asesora de Pensiones de la Confederación de la ProDUCCIÓN Y EL COMERCIO, Informe de pensiones, 2017.

COMISIÓN MARCEL, 2006, disponible en: https:// wmm.previsionsocial.gob.cl/sps/ download/estudios-previsionales/comisionpensiones/documentos-interes-general/ informe-consejo-asesor-presidencial-reforma-del-sistema-previsional-comision-marcel-2006.pdf.

Cunill-Grau, Nuria y LeYTON, Cristian, "Una nueva mirada al enfoque de derechos. Aportes al debate a partir de los servicios de cuidado para personas mayores en Chile", Revista Latinoamericana de Derecho Social, núm. 27, 2018.

Esping-Andersen, Gosta, Three Worlds of Welfare Capitalism, Princeton, New Jersey, Princeton University Press, 1990.

FuENTES-GARCíA, Alejandra et al., "Socioeconomic Inequalities in the Onset and Progression of Disability in a Cohort of Older People in Santiago", Gaceta Sanitaria, Chile, 2013.

GUMUCIO, Juan, "Una mirada al sistema de pensiones de adminstración privada chileno al acercarse un cuarto de siglo de aplicación", Revista Latinoamericana de Derecho Social, núm. 2, enero-junio, 2006.

Hojman, Daniel et al., "The Cost of Dementia in an Unequal Country: The Case of Chile", PloS One, 2017.

Huenchuan, Sandra et al., Protección y participación en la vejez: escenarios futuros y políticas públicas para enfrentar el envejecimiento en Chile, Santiago de Chile, CEPAL, 2007.

Instituto NACiOnal de Estadísticas, Encuesta Nacional de Empleo, Santiago de Chile, Instituto Nacional de Estadísticas, 2017.

Instituto NACional DE Estadísticas, Encuesta suplementaria de ingresos 2017, Santiago de Chile, Instituto Nacional de Estadísticas, 2018.

KORPI, Walter y PALME, Joakim, "The Paradox of Redistribution and Strategies of Equality: Welfare State Institutions, Inequality, and Poverty in the Western countries", American Sociological Review, 1998.

LANATA, Gabriela, Manual de legislación previsional, Santiago de Chile, Thomson Reuters, 2015.

MADERO-CABIB, Ignacio y FASAng, Anette, "Gendered Work-Family Life Courses and Financial Well-Being in Retirement", Advances in Life Course Research, 2016. 
MADERO-CABIB, Ignacio, Regimenes de pensiones con bases laborales precarias. Lecciones de un estudio longitudinal en Chile. Presentación en seminario "Edad, trabajo y pensiones. Viejos y nuevos retos", Flacso Chile-Centro de Estudios de Derecho del Trabajo y Seguridad Social de la Universidad de Talca, Santiago de Chile, 2018a.

Madero-CABIB, Ignacio, Proyecto Fondecyt Postdoctorado núm. 3160522, "The impact of employment trajectories and retirement transition timing on older adults' health: A longitudinal study in 11 countries", 2018b.

NAZAR, Gabriela y FigueroA, Carolina, "Creencias estereotípicas sobre el desempeño laboral de trabajadores mayores en Chile", Psicoperspectivas, 2015.

OCHANDO, Carlos, El Estado del bienestar: objetivos, modelos y teorías explicativas, Madrid, Ediciones Académicas, 2009.

OECD (2012), OECD pensions outlook 2012, Paris, OECD Publishing.

OECD (2013), Pensions at a Glance 2013, OECD and G20 Indicators, Paris, OECD Publishing.

OECD (2015), Pensions at a Glance 2015, OECD and G20 indicators, Paris, OECD Publishing.

OECD (2017), Labour force participation rate (indicator), doi: 10.1787/8a801325-en (Accessed on 13 October 2018).

OECD (2018), Gross pension replacement rates (indicator), doi: 10.1787/3d1afeb1-en (Accessed on 16 October 2018).

OIT (2014), Social protection for older persons: key policy trends and statistics.

OIT (2011), La igualdad en el trabajo: un objetivo que sigue pendiente de cumplirse. Informe global con arreglo al seguimiento de la Declaración de la OIT relativa a los principios y derechos fundamentales en el Trabajo, Conferencia Internacional del Trabajo, $100^{\circ}$ reunión, Ginebra, OIT.

PALOMO, Rodrigo y SALAZAR, Fernanda, "Acuerdo marco autónomo sobre envejecimiento activo y enfoque intergeneracional en la Unión Europea", Revista Laboral Cbilena (julio), Santiago de Chile, Ediciones Jurídicas Ltda, 2017.

PIÑERA, José, El cascabel al gato: la batalla por la reforma previsional, Santiago, Chile, Zig-Zag, 1991. 
Programa Adulto Mayor UC Chile y sus Mayores, Resultados Tercera Encuesta Nacional Calidad de Vida en la Vejer, Santiago de Chile, Pontificia Universidad Católica de Chile-Caja Los Andes, 2013.

RojAs, Irene, Derecho del trabajo. Derecho individual del trabajo, Santiago de Chile, Thomson Reuters, 2015.

Rotarou, Elena y SAKELlariou, Dikaios, "Inequalities in Access to Health Care for People with Disabilities in Chile: the Limits of Universal Health Coverage", Critical Public Health, 2017.

THumala, Daniela et al., Inclusión y exclusión social de las personas mayores en Chile, Santiago de Chile, Ediciones Servicio Nacional del Adulto Mayor, Senama-FACSO Universidad de Chile, 2015.

Thumala, Daniela et al., "Aging and Health Policies in Chile: New Agendas for Research", Health Systems y Reform, 2017.

UNDP, Desiguales. Orígenes, cambios y desafíos de la brecha social en Chile, Santiago de Chile, Programa de las Naciones Unidas para el Desarrollo, 2017.

VIVES, Alejanra et al., "Envejecimiento y trabajo en Chile: propuesta para el monitoreo de la salud laboural", Propuestas para Chile (concurso políticas públicas), Santiago de Chile, Pontificia Universidad Católica de Chile, 2016.

ZITKO, Pedro y CABIESES, Baltica, "Socioeconomic Determinants of Disability in Chile", Disability and Health Journal, 4(4), 2011. 